\title{
High Frequency of AIFM1 Variants and Phenotype Progression of Auditory Neuropathy in a Chinese Population
}

\author{
Hongyang Wang, ${ }^{1,2}$ Dan Bing, ${ }^{3}$ Jin Li, ${ }^{1,2}$ Linyi Xie, ${ }^{1,2}$ Fen Xiong, ${ }^{1,2}$ Lan Lan, ${ }^{1,2}$ \\ Dayong Wang, ${ }^{1,2}$ Jing Guan, ${ }^{1,2}$ and Qiuju Wang $\mathbb{D}^{1,2}$ \\ ${ }^{1}$ College of Otolaryngology, Head and Neck Surgery, Chinese PLA Institute of Otolaryngology, Chinese PLA General Hospital, \\ Beijing 100853, China \\ ${ }^{2}$ National Clinical Research Center for Otolaryngologic Diseases, Beijing 100853, China \\ ${ }^{3}$ Department of Otolaryngology-Head and Neck Surgery, Tongji Hospital, Tongji Medical College, Huazhong University of Science \\ and Technology, Wuhan 430030, China
}

Correspondence should be addressed to Qiuju Wang; wqcr301@vip.sina.com

Received 6 March 2020; Revised 14 May 2020; Accepted 2 June 2020; Published 1 July 2020

Academic Editor: Renjie Chai

Copyright (c) 2020 Hongyang Wang et al. This is an open access article distributed under the Creative Commons Attribution License, which permits unrestricted use, distribution, and reproduction in any medium, provided the original work is properly cited.

\begin{abstract}
To decipher the genotype-phenotype correlation of auditory neuropathy (AN) caused by AIFM1 variations, as well as the phenotype progression of these patients, exploring the potential molecular pathogenic mechanism of AN. A total of 36 families of individuals with AN (50 cases) with AIFM1 variations were recruited and identified by Sanger sequencing or next-generation sequencing; the participants included 30 patients from 16 reported families and 20 new cases. We found that AIFM1-positive cases accounted for $18.6 \%$ of late-onset AN cases. Of the 50 AN patients with AIFM1 variants, 45 were male and 5 were female. The hotspot variation of this gene was p.Leu344Phe, accounting for $36.1 \%$. A total of 19 AIFM1 variants were reported in this study, including 7 novel ones. A follow-up study was performed on 30 previously reported AIFM1-positive subjects, 16 followup cases $(53.3 \%)$ were included in this study, and follow-up periods were recorded from 1 to 23 years with average $9.75 \pm 9.89$ years. There was no hearing threshold increase during the short-term follow-up period (1-10 years), but the low-frequency and high-frequency hearing thresholds showed a significant increase with the prolongation of follow-up time. The speech discrimination score progressed gradually and significantly along with the course of the disease and showed a more serious decline, which was disproportionately worse than the pure tone threshold. In addition to the X-linked recessive inheritance pattern, the X-linked dominant inheritance pattern is also observed in AIFM1-related AN and affects females. In conclusion, we confirmed that AIFM1 is the primary related gene among late-onset AN cases, and the most common recurrent variant is p.Leu344Phe. Except for the X-linked recessive inheritance pattern, the X-linked dominant inheritance pattern is another probability of AIFM1-related AN, with females affected. Phenotypical features of AIFM1-related AN suggested that auditory dyssynchrony progressively worsened over time.
\end{abstract}

\section{Introduction}

Auditory neuropathy (AN) is a special type of sensorineural hearing loss with a main manifestation of impaired speech comprehension, accounting for $1.2-10 \%$ of cases of hearing loss, depending on the population $[1,2]$. The affected hearing in AN is mainly low frequency, and the speech recognition rate is obviously disproportionately lower than the pure tone threshold (PTA). This type of disease may arise from the inner hair cells (IHCs) of the cochlea, the synapses between the IHCs and the auditory nerve, the spiral ganglion neuron (SGN), the cochlear nerve fibers, and one or more of the auditory nerves [3].

The pathogenic mechanism of AN is currently unclear, and genetic factors may account for up to $40 \%$ of the pathogenesis of AN [4]. The inheritance pattern of AN includes autosomal recessive, autosomal dominant, and X-linked recessive inheritance. In 2006, our group located the gene 
locus AUNX1 of X-linked recessive hereditary neuropathy in the Xq23-q27.3 region for the first time [5] and then further identified AIFM1 as the gene responsible for this kind of AN using whole exome sequencing technology in 2015 [6]. Apoptosis-inducing factor (AIF) is a flavin protein that is located in the mitochondrial membrane space. It was originally discovered as the first apoptotic factor that causes caspase-independent apoptosis [7]. This protein plays a critical role in maintaining the normal morphology and physiological functions of mitochondria and causing apoptosis that is not dependent on caspase.

Since AN was first identified more than 20 years ago, diagnosis, particularly precision diagnosis with lesion site identification, remains a challenge. Cases with genetic basis and the identification of the genes may be helpful for the lesion site identification, deciphering the underlying mechanism of AN [8-9]. Except for diagnosis, intervention is another challenge for clinical management for AN. Hearing aids and cochlear implantations, which are typical intervention strategies for cochlear sensory hearing loss, have variable outcomes for AN cases depending on the affected lesion sites [3-10]. Gene therapy may provide possibility for the treatment of AN [11]. Since virally mediated gene expressions in almost $100 \%$ HCs are possible, the treatment of presynaptic $\mathrm{AN}$ is possible [12]. And the virally expressing genes in SGNs are also feasible, supporting the possibility of treating postsynaptic AN [13].

Up to date, there is no frequency data of AIFM1-positive cases in AN cases. In this study, we further identified another 20 AN cases with AIFM1 variants, including 7 novel variants and one hotspot variant, showing that the proportion of $\mathrm{AN}$ caused by AIFM1 in Chinese patients with delayed-onset AN was as high as $18.6 \%$ (36/194), higher than the $15.53 \%$ $(16 / 103)$ observed in the previous study [6]. Genotypephenotype correlation analyses of AN cases with AIFM1 gene pathogenic mutations were carried out, including the clinical hearing vestibular test, comprehensive clinical follow-up study data, and an in-depth exploration of the clinical characteristics of the disease and the related pathogenesis, to explore the characteristics of AIMF1 gene-positive $\mathrm{AN}$, laying the theoretical basis of AN classification diagnosis. The X-linked dominant inheritance pattern is also firstly observed in AIFM1-related AN and affects females in the study as well.

\section{Materials and Methods}

2.1. Ethics Statement. The study was approved by the Committee of Medical Ethics of Chinese PLA General Hospital. Written informed consent was obtained from all participants.

2.2. Subject Recruitment and Clinical Evaluation. A total of 50 patients with AIFM1 mutations who were diagnosed with AN in the Chinese PLA Institute of Otolaryngology, Chinese PLA General Hospital, from April 1997 to June 2019 were recruited for this study. The diagnostic criteria were as follows: The typical audiological characteristics were that the auditory brainstem response (ABR) had no obvious differen- tiation waveform or severe abnormality and that the otoacoustic emission (OAE) and/or the cochlear microphonic (CM) potential could be normally extracted. Personal or family medical evidence of hearing loss, tinnitus, vestibular symptoms, and other clinical abnormalities of both the affected members and the unaffected members of these families was identified. Pure tone threshold (PTA), speech discrimination score (SDS), ABR, OAE, CM, and electrocochleography (ECochG) were carried out as otological examination batteries to evaluate auditory status. In general, the low frequencies were primarily affected; thus, we focused on the low-frequency data and calculated PTA as the average of the thresholds of $250-1000 \mathrm{~Hz}$ to avoid bias in the assessment of the degree of AN hearing loss. Vestibular function evaluation included vestibular evoked myogenic potentials, oculomotor function tests, positional nystagmus tests, positioning nystagmus tests, and bithermal caloric tests. Highresolution computed tomography (CT) scans of the temporal bone and cerebral magnetic resonance imaging (MRI) were performed to exclude other possible neuropathic or anatomical disorders.

2.3. Genetic Techniques. Next-generation sequencing and Sanger sequencing were performed on the patients as previously described. Variation interpretation (evaluation of the pathogenicity) was based on the standards and guidelines of the American College of Medical Genetics and Genomics and the Association for Molecular Pathology (ACMG and AMP) [14].

2.4. Statistical Analysis. Statistical analysis was performed using SPSS 19.0 statistical software, Empower software (http://www.empowerstats.com, X\&Y Solutions, Inc., Boston, MA) and R software (https://www.R-project.org), as well as G. Comparisons of the two sets of data were performed using an independent sample $t$-test. The comparisons of multiple sets of data were performed using one-way ANOVA. $p<0.05$ represented a significant difference. Spline smoothing was performed using GAMM (generalized additive mixed model) to explore the change in pure tone threshold with the length of follow-up time.

\section{Results}

3.1. General Clinical Information. Fifty patients with AIFM1 mutations were recruited for this study, including 30 patients who have been previously studied and 20 novel patients who were identified recently (Table 1 ). All patients had no history of high-risk factors such as hyperbilirubinemia and hypoxia and denied a history of metabolic diseases such as diabetes. Nine (18\%) patients had a history of ototoxic drug use, and one patient had a history of exposure to noise. Clinically, 37 patients $(74 \%)$ complained of tinnitus at the first visit, and numbness of the extremities was the second most common symptom (12 patients), while few patients experienced visual impairment (10 patients) and vertigo (8 patients). Fifteen patients (30 ears) received cVEMP examination, among which $19(63.3 \%)$ ears showed ipsilateral sacculus dysfunction, and 11 (36.7\%) ears showed normal function. Nineteen 


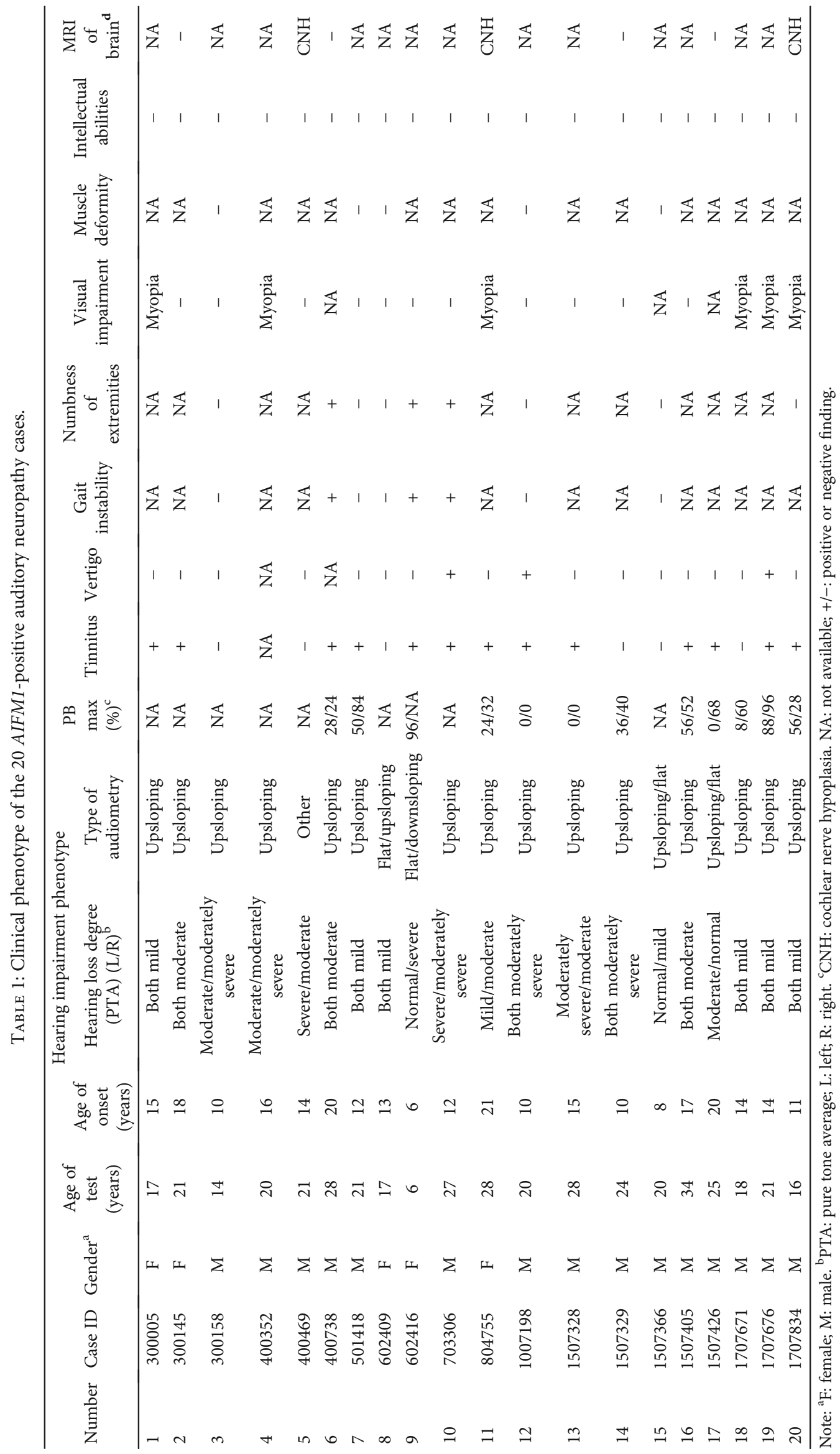


patients underwent vestibular function tests; 15 sides $(15 / 38$, $39.5 \%$ ) showed abnormalities, while the other 23 sides were normal. No abnormalities in temporal CT were found, and 3 patients $(42.9 \%, 3 / 7)$ showed bilateral cochlear nerve hypoplasia in the MRI test.

The pure tone thresholds of each frequency were not significantly different between the left and right ears in all 50 patients (Figure $1(\mathrm{a})$ ). The disease durations at the first visit ranged from 0 to 33 years $(9.7 \pm 7.7$ years), and the PTAs among the 5-, 5- to 15-, and >15-year disease duration groups were not significantly different $(p>0.05)$ (Figure 1(b)). The onset ages of AIFM1-positive patients ranged from 5 to 20 years $(13.4 \pm 3.9)$. In addition, the earlier the onset, the more severe the hearing impairment. The pure tone averages (PTAs, 250-1000 Hz) of the <12-year-old group, 12- to 16year-old group, and >16-year-old group were 55.7, 51.2, and $47.7 \mathrm{~dB} \mathrm{HL}$, respectively. The most severe hearing loss was observed in the youngest age group. However, the differences in hearing loss between the three onset age groups were not statistically significant (Figure 1(c)).

3.2. Mutation Spectrum of AIFM1 (Table 2). Among the 20 novel cases found with AIFM1 variations, seven novel and three reported variations were found, located in the FAD, $\mathrm{NADH}$, and C-terminus. Pathogenicity was assessed using SIFT (http://sift.jcvi.org/), PolyPhen-2 (Polymorphism Phenotyping V.2, http://genetics.bwh.harvard.edu/pph2), LRT (http://www.genetics.wustl.edu/jflab/lrt_query.html), and MutationTaster (http://www.mutationtaster.org).

The seven novel variations were all pathogenic with the evidences of "PS $\times 1+\mathrm{PM} \times 2+\mathrm{PP} \times 2$ " according to the ACMG and AMP guidelines [14]. Firstly, $18.6 \%$ of late-onset AN cases had variants in AIFM1, while the variations were absent in our sensorineural hearing loss group (PS4). Secondly, these variations were absent or at extremely low frequency in ESP, ExAC, gnomAD-EAS, or 1000genomes (PM2). Thirdly, in these families, the variations were cosegregated with $A N$ phenotype, with proband's mother carrying variation but having no AN performance (PM). Fourthly, these variants were predicted to be deleterious with SIFT, PolyPhen-2, LRT, MutationTaster, and so on (PP3). In addition, in terms of phenotype, these patients were all reported as AN(PP4). Furthermore, cells with the AIFM1 mutation led to decreased dimerization and impaired mitochondrial functions (unpublished data), which may indicate that the mutations in AIFM1 gene may affect auditory function, providing PS3 evidence.

The recurrent variants were p.Ile304Met, p.Leu344Phe, p.Arg422Trp, and p.Tyr560His, among which the most common variant in the AN population was p.Leu344Phe, which was present in $36.1 \%(13 / 36)$ of the positive cases, followed by p.Arg422Trp $(13.9 \%, 5 / 36)$.

In total, 18 variations in $A I F M 1$ variations were related to the AN phenotype, with 9 variations located in FAD, 6 variations in $\mathrm{NADH}$, and 4 variations in the C-terminal region. There was no overlap with the other AIFM1 variations that caused other syndromes [6, 15-28] (Figure 2).

\subsection{Genotype and Phenotype Correlation Analysis of the 20 Newly Identified Cases}

3.3.1. Clinical Features of the 20 Newly Identified Cases with AIFM1 Variants (Table 1). Except for families 0804755 and 1507328, who had a family history, 18 other cases with AIFM1 variants were sporadic cases. The age of onset ranged from 6 to 20 years, with only one case (1507426) not complaining of childhood-onset AN. Except for one female case with unilateral $\mathrm{AN}$, all other patients showed bilateral AN. The audiograms varied, with 31 ears $(83.8 \%, 31 / 37)$ showing upsloping types. Seven of 20 patients underwent inclined sagittal MRI of the internal auditory canals, with 3 patients showing bilateral cochlear nerve hypoplasia.

3.3.2. Female Patients with AIFM1 Variants (Possible XLinked Dominant Inheritance Pattern). All of the female cases had the same variant, c.1030C > T (p.Leu344Phe), which was also the most common variant among AN-related AIFM1 variations. Except for 0804755 (Figure 3(a)), the other female patients had no family history. There was no hearing threshold difference between male and female AN patients with this variant (Figure 3(b)). However, the audiograms of the females affected varied from normal to profound, including flat, upflopping, and downflopping as time went on (Figure 3(c)).

3.3.3. Phenotype Follow-Up of the 30 AN Patients with AIFM1 Mutations. Sixteen patients (53.33\%) underwent follow-up pure tone audiometry tests, and the follow-up period was 1-23 years, with a mean time of $9.75 \pm 9.89$ years. The hearing threshold change varied between patients (Figure 4). The average thresholds of low and high frequencies in the different follow-up groups were significantly different in all of the patients who had been followed for more than one year (Figure 1(d)). For the patients with a short-term follow-up period (1-10 years), the hearing deterioration was not apparent at all frequencies (Figure 1(e)), but with the prolongation of follow-up time (more than 10 years), the low-frequency $(0.25$ and $0.5 \mathrm{kHz})$ and high-frequency ( 4 and $8 \mathrm{kHz}$ ) hearing thresholds showed a significant increase (Figure 1(f)).

In addition, we performed spline smoothing by using GAMM to explore the change in the pure tone threshold with the length of follow-up time. Figure 5 illustrates the shape of the relationship between the hearing outcome at a frequency of $0.5 \mathrm{kHz}$ and the follow-up time (edf $=1.427, p=0.0096$ ). This result suggested that the hearing threshold of $0.5 \mathrm{kHz}$ in both ears worsened gradually over time. The hearing thresholds of $0.25,1,2,4$, and $8 \mathrm{kHz}$ changed in a similar pattern as that of $0.5 \mathrm{kHz}$, although statistical significance was not reached in either ear (Figure 6).

Twenty-three patients (46/60 ears, $76.67 \%)$ with AIFM1 mutations underwent binaural speech testing, and 10 patients (20 ears, 43.5\%) were followed up. A total of 10 patients (20 ears) were followed up for SDS (Table S1), with a follow-up period of $1-15$ years $(6.80 \pm 4.47$ years $)$. Of the 46 ears, $50 \%$ of patients with mild hearing loss had zero SDS, which was a much higher figure than that in the moderate and severe groups. Overall, the SDS of the mild hearing loss group was significantly lower than that of the moderate and severe groups $(p<0.05)$. Therefore, the degree of SDS decline in these patients with AN was not 


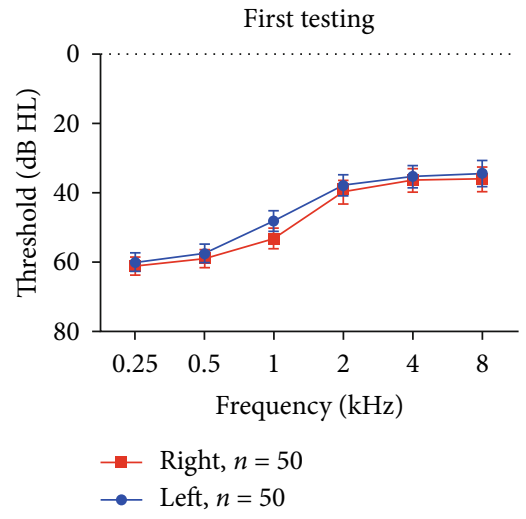

(a)

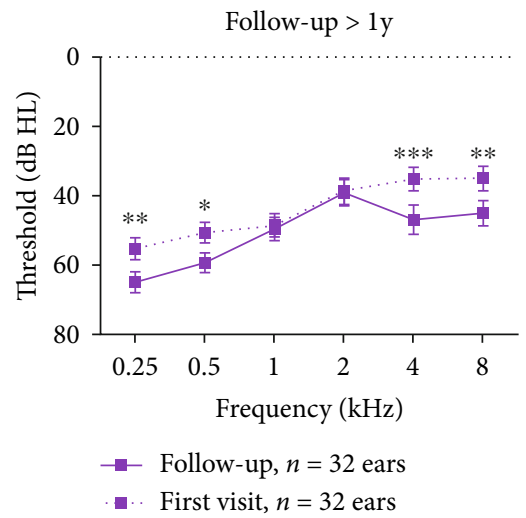

(d)

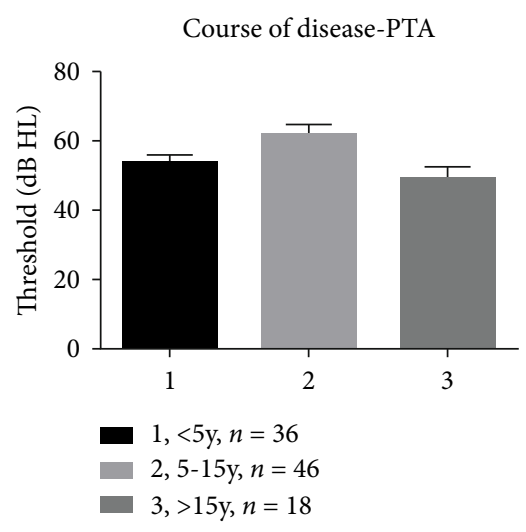

(b)

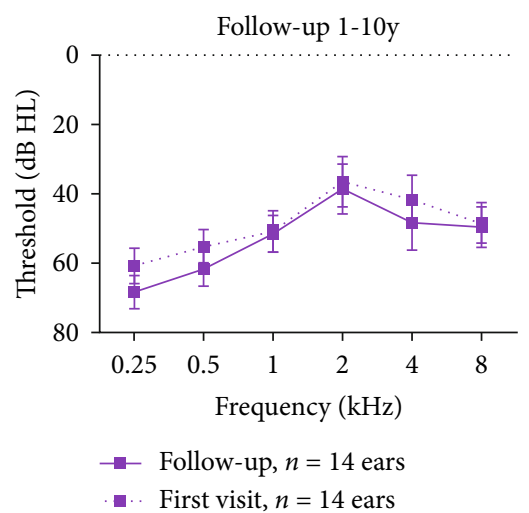

(e)

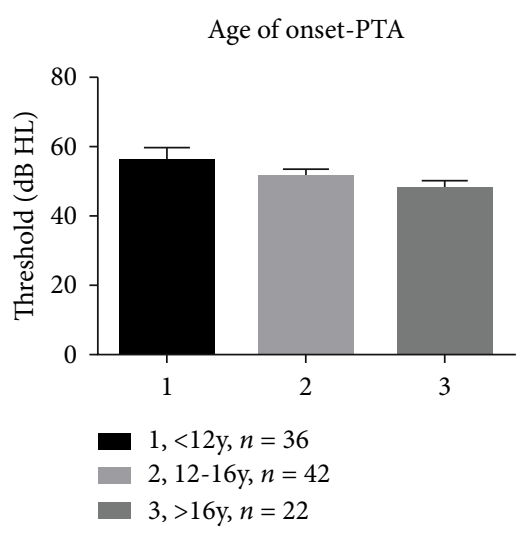

(c)

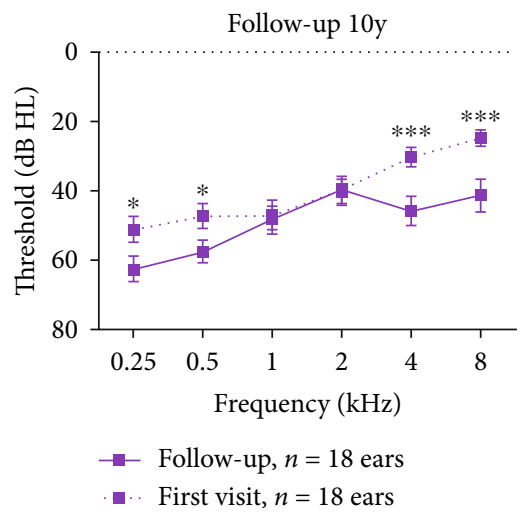

(f)

FIgure 1: Pure tone test of the AIFM1-positive cases. (a) Average threshold of the 50 cases with AIFM1 variations. (b) Mean PTA in the groups with different disease courses. (c) Mean PTA in the groups with different onset ages. (d-f) Mean threshold in each frequency with different follow-up periods. dB: decibels; Hz: Hertz; PTA: pure tone average.

proportional to the pure tone hearing threshold, and SDS was more severe in patients with mild hearing loss than in patients in the moderate and severe hearing loss groups (Table S2). Disease duration was another risk factor; of the 9 patients with a disease duration less than 5 years, none had an SDS score of zero. In patients with disease duration of more than 5 years, the proportion of patients with an SDS score of zero increased significantly, accounting for $50 \%$ of all ears (Table S3). This finding indicates that SDS decreased significantly with the prolongation of the disease course, and the difference was statistically significant $(p<0.05)$. The mean values of the left and right ears before and after follow-up were greater than zero, indicating that the SDS exhibited a downward trend before and after follow-up, but the difference between the two ears was not statistically significant $(p>0.05)$.

Among the 30 patients, 7 ears from 5 patients were able to elicit $\mathrm{V}$-waves, but the waveform differentiation was poor, the amplitudes were reduced, and the latencies were prolonged. The PTAs of the 7 ears with the V-wave were relatively better, and the course of the disease was shorter than that of the unexposed ABR waveform, but the difference was not statistically significant $(p>0.05)$ (Table S4). Three of five patients had follow-up ABR data; their $\mathrm{V}$-wave latencies were gradually extended, and the $\mathrm{V}$-wave of $\mathrm{ABR}$ was unextracted in the follow-up of one ear (left ear of 1007170-1) (Table S5).

All 30 patients underwent DPOAE; 29 patients passed with the elicitation of at least five frequencies, while one patient had no response at any of the frequencies. This individual was a member of family 1007170 and had a disease duration of 33 years. AN may progress to sensorineural hearing loss, with outer hair cell impairment as time goes on.

A total of 22 patients (44 ears) underwent electrocochleography examination, among whom, 6 patients underwent follow-up observation. The -SP waves were found in 21 patients, except for one person who had an unobvious wave. Nine patients $(20.5 \%, 9 / 44)$ showed the -SP wave only, without an obvious CAP wave, while the remaining 35 ears showed both -SP and CAP waveforms, with absolute values of -SP/AP > 0.4 (Table S6). The degree of hearing loss in patients with AN who did not elicit CAP waveforms was significantly higher than that in patients with CAP, and the difference was statistically significant $(p<0.001)$. For the 6 cases (12 ears) with a follow-up ECochG test (Figure S1), there were no differences between the absolute values of SP/AP. 


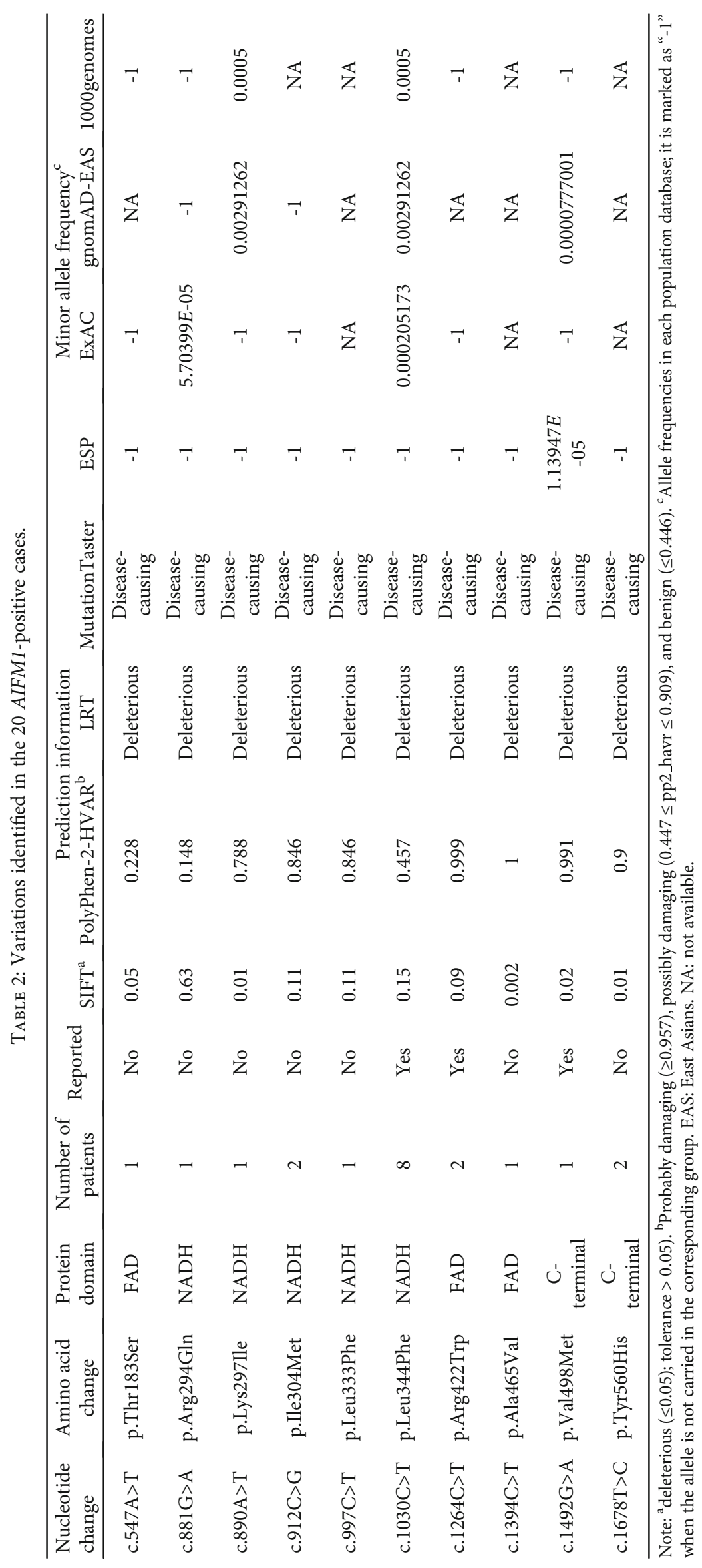




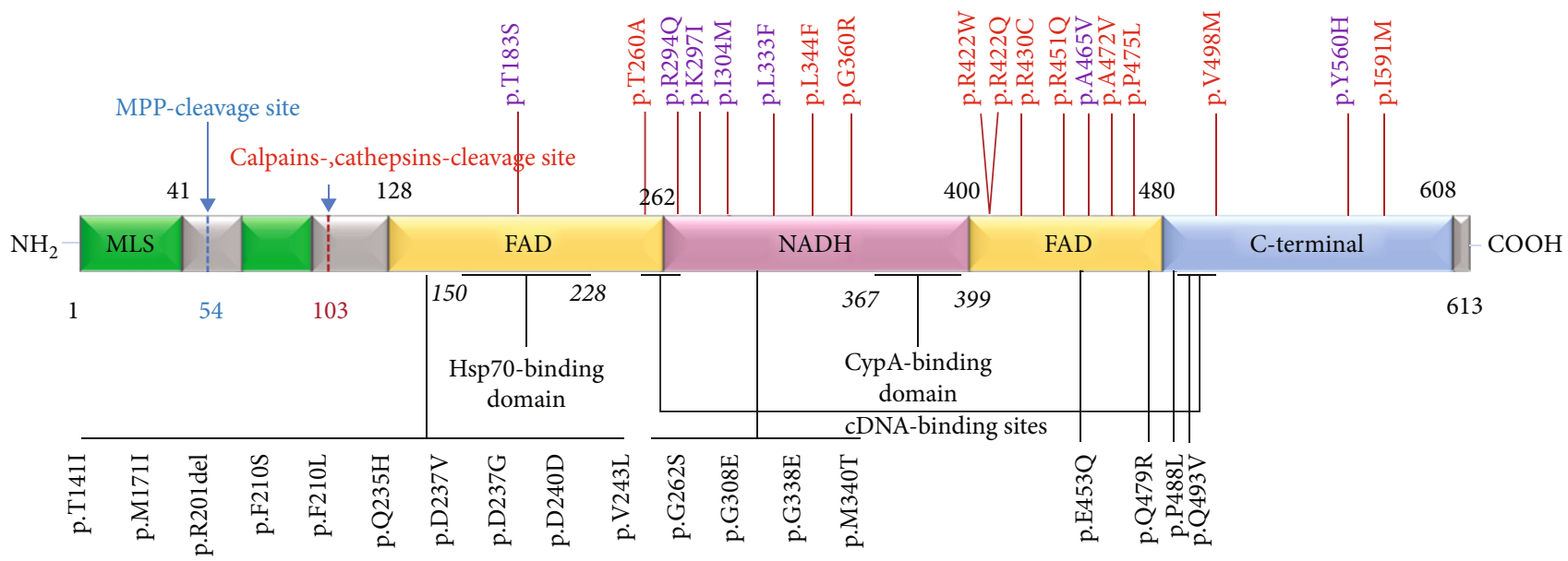

FIGURE 2: AIFM1 variations in auditory neuropathy as well as other syndromes. The purple ones are the new variations identified in this study, the red ones are the previously reported AN-related variations, and the black ones are the variants that are related to syndromes such as cerebellar ataxia (modified from [6]).

\section{Discussion}

AN is a special type of hearing dysfunction disease, which is one of the critical diseases that cause speech communication disorders in infants and adolescents [3]. In the auditory system, HCs and SGNs are very important for hearing ability; HCs convert the sound waves into electrical signals, and SGN transmit the electrical signals into the auditory cortex for hearing ability [29]. In a mammal's cochlea, HCs and SGNs are vulnerable for multiple damages, including noise, gene mutation, ototoxic drugs, inflammation, and aging [30-34]; while the mammal's cochlea only have very limited HC and SGN regeneration ability, majority of the damaged HC and SGN cannot be spontaneously regenerated [3541]. Thus, hearing loss is usually irreversible, and AN may come from the damage of IHC and SGNs.

As a difficult and popular topic in international research, research on $\mathrm{AN}$ has been performed for 20 years, from preliminary reports to various explorations of its pathogenesis, and it is beginning to be gradually understood accurately. The etiology of AN varies with age, genetic factors, hyperbilirubinemia, low birth weight, premature birth, and hypoxia. More patients may be discovered as the use of genetic testing in the diagnosis of auditory neuropathy becomes more widespread [4-8]; however, no prevalence studies have been performed to date. In our previous study, we confirmed that OTOF is the most common gene-causing congenital auditory neuropathy [42]. In contrast, for patients with late-onset AN, the etiology varies and is associated with optic atrophy, sensorimotor neuropathy, and other peripheral neuropathies. Among the late-onset cases, AIFM1 is reported to be the most common genetic cause [6]. In this study, we further confirmed that AIFM1 is the most common genetic cause of all noninfant-onset AN cases. The identification of genes is helpful to identify related lesion sites of AN and contributes to a better understanding of the underlying pathogenic mechanisms $[8,9]$.

The AIFM1 gene, also known as AIF, PDCD8, COXPD6, etc., is located in the human chromosome Xq25-q26 region, with a full length of $36.471 \mathrm{~kb}$ and 16 exons encoding a fulllength 613 amino acid protein. In the mitochondria, AIFM1 acts as a FAD-dependent NADH oxidoreductase and plays a critical physiological role in the stable and mature mitochondrial oxidative respiratory chain complex I and the elimination of peroxide. In this study, we further expanded the mutation spectrum and long-term phenotypes of AIFM1related cases. We found another $20 \mathrm{AN}$ cases with AIFM1 variants by whole genome sequencing and Sanger sequencing, including 7 novel variants. All $18 \mathrm{AN}$-related variations had no overlap with the other phenotype-related AIFM1 variations (Table S7). In addition, we confirmed that the most common variation is AIFM1 c.1030C $>\mathrm{T}$ (p.Leu344Phe). Due to its location in the loop region, this variation may have an influence on folding.

In this study, we found 5 female AN cases with AIFM1 variants, the phenotype of whom was similar to those of the male cases with the same variant. AIFM1 gene mutation AN may also be inherited in an X-linked dominant inheritance pattern. In our previous study, except for the patients undergoing whole exome sequencing, we did not pay attention to female AN cases; only male cases were tested for the AIFM1 gene.

To decipher the phenotype progression of AIFM1-related AN, a follow-up study was performed. Further genotypephenotype correlation analysis can effectively help physicians and patients understand the disease-causing mechanism, process, and outcome of disease by research methods. To further assist in the consultation and evaluation of prognosis in patients with clinical AN, the clinical phenotypes of AIFM1related cases were as follows:

(1) For the pure tone threshold, AN patients have large individual differences in audiologic phenotype. Although the ascending audiogram is the typical audiometric pattern of AN patients, audiograms with various configurations and varying severities may occur. The hearing of some patients may improve, while others' hearing loss may remain stable for a 

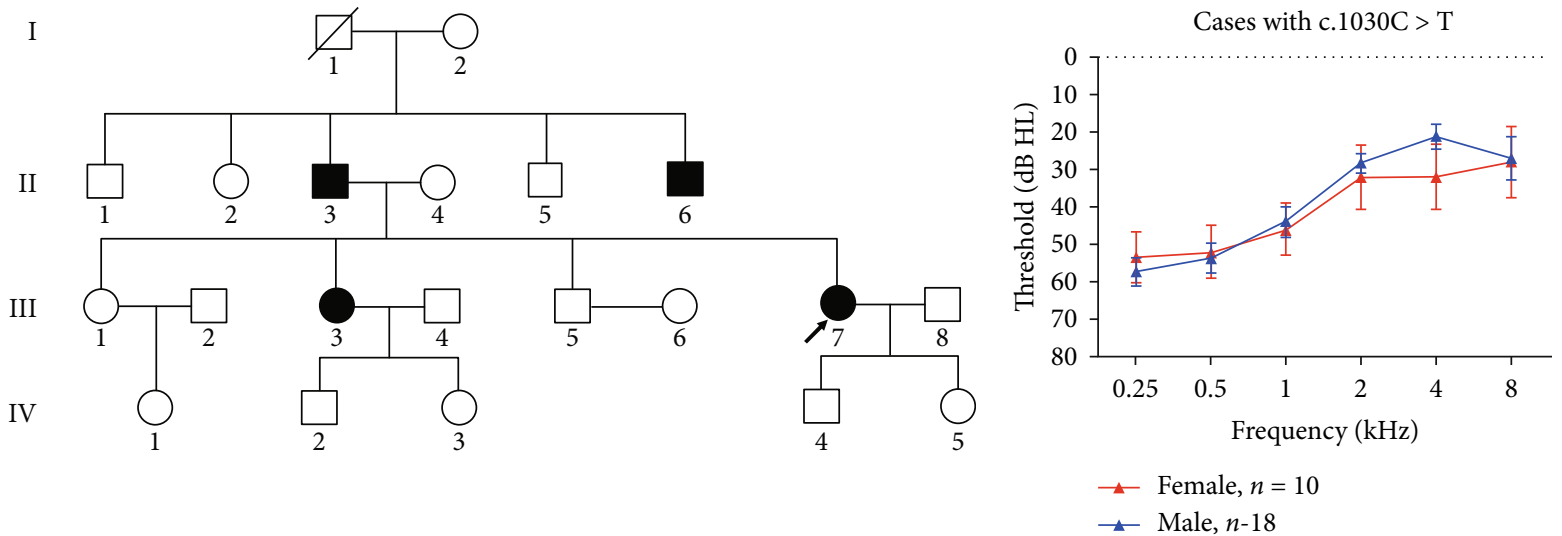

(a)
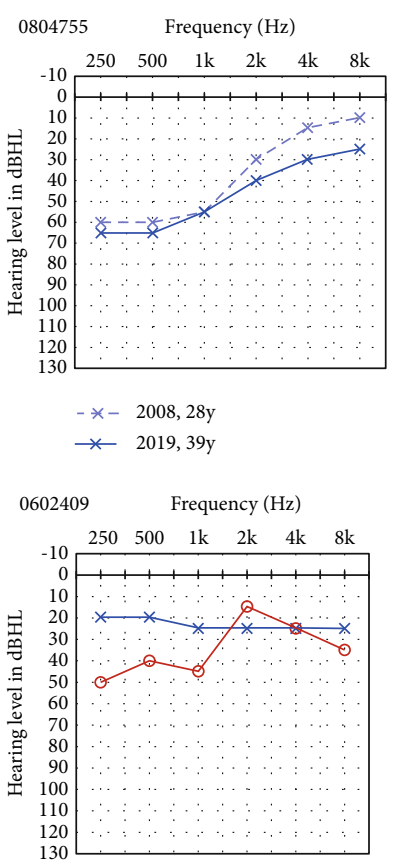
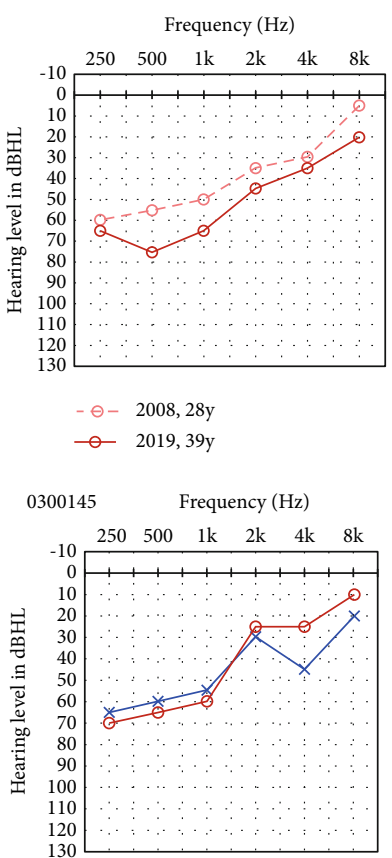
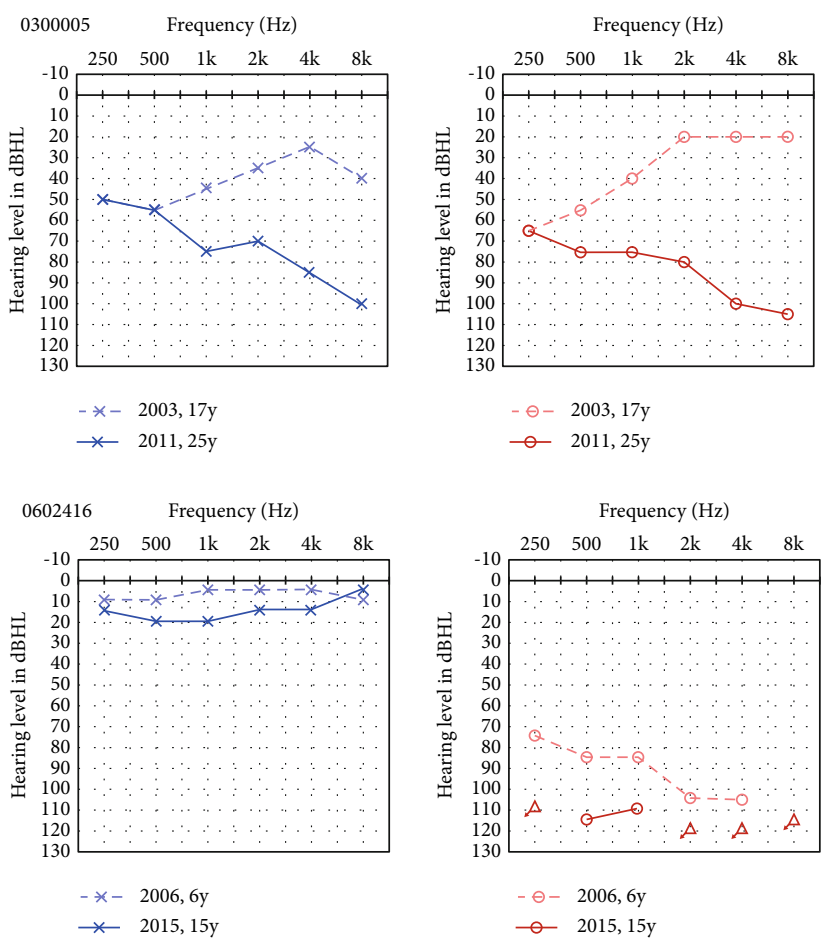

(b)

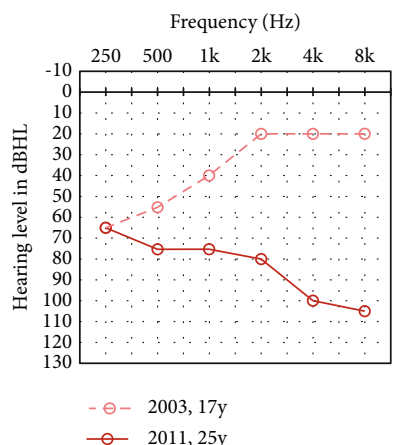

- $2015,15 y$

(c)

Figure 3: Family trees and audiological characteristics of the five female cases. (a) Family trees of the family 0804755. (b) Mean hearing threshold of the cases with the AIFM1 c.1030C > T (p.Leu344Phe) variation. (c) Audiograms of the five female affected cases. Symbols "o" and " $\mathrm{x}$ " denote air conduction pure tone thresholds at different frequencies in the right and left ears. dB: decibels; Hz: Hertz. The dashed line represents the audiograms detected in the first time, while the solid lines were the latest audiological examinations. y: years old.

long time or even worsen. Among the very limited reports involving the follow-up characteristics of AN patients, the hearing outcomes in a long-term follow-up remain elusive [43]. Not surprisingly, we found, in the 50 AIFM1-positive cases, that the low frequency spectra were mostly affected, especially in the $0.25-1 \mathrm{kHz}$ range. Hearing impairment ranged from mild to moderate. No significant differences were detected in the hearing thresholds tested between the first and final visits within 1-10 years of follow-up. However, when the follow-up periods were prolonged to over ten years, hearing thresholds in both the low frequencies $(0.25-0.5 \mathrm{kHz})$ and the high frequencies $(4-8 \mathrm{kHz})$ showed significant worsening. In addition, the pure tone threshold tended to deteriorate over time, especially at a frequency of $500 \mathrm{~Hz}$

(2) For SDS, the AN patient's prominent complaint is that he/she can hear voices without understanding the meaning. Our study found that patients with mild hearing loss had a high proportion of a score of zero when testing SDS, which was much more severe than the SDS in the moderate and severe hearing loss groups, and the degree of SDS was not consistent with the pure tone threshold. This fact suggests that 

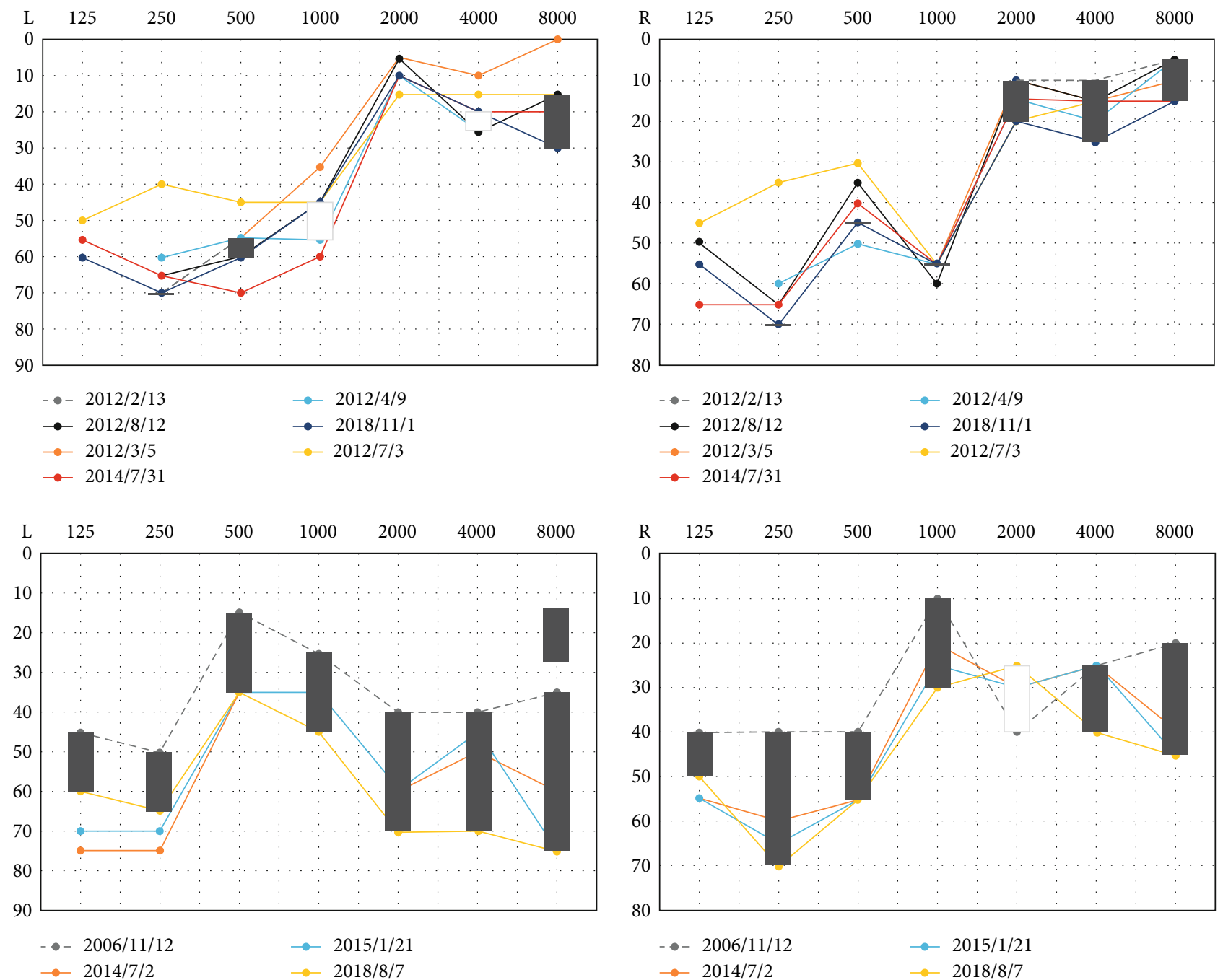

FIgURE 4: Typical cases with stable and progressive pure tone hearing thresholds.

in clinical audiology assessment, the SDS performance of AN patients is much more critical than that of PTA. The evaluation of the degree of hearing loss by PTA alone may underestimate the patient's condition. Furthermore, we followed up on the SDS of this type of AN patient and found that SDS decreased significantly with the prolongation of the disease course. This result indicates that the neurological synchronization of the AIMF1 gene-related AN is gradually aggravated

(3) ECochG revealed that -SP and CAP waves existed together in $80 \%$ of cases, but the SP/AP values were higher than normal. Patients showed worse hearing loss when their CAP waves disappeared. Compared with DPOAE, cochlear electrograms can help us locate the lesions of AN [44]. The -SP wave reflects the fractional depolarization process after the inner hair cells are subjected to the acoustic signal and is the maximum amplitude recorded by the needle electrode placed on the cochlear or round window through the tympanic membrane [45]. The -SP waves are mainly derived from inner hair cells, and their amplitude and latency are objective indicators of the function of inner hair cells. Except for one patient without obvious one-time single-SP, the -SP wave of all of the other AN patients could be seen, suggesting that the AN lesion caused by AIFM1 gene mutation may be located in the auditory conduction pathway outside the inner hair cells. CAP is produced in cochlear spiral ganglion cells and is an afferent nerve response. The decrease in CAP amplitude can prove the synchrony decline in auditory nerve activity. We observed that the -SP and CAP waveforms were present in $80 \%$ of patients at the same time, but the absolute value of -SP/AP was $>0.4$, which was higher than normal, suggesting that there was a loss of synchronization of auditory nerve activity. This also explains why the SDS decline in this type of patient is more marked than the decrease in PTA from the perspective of physiology and pathology. Furthermore, we analyzed the patient's auditory condition based on 


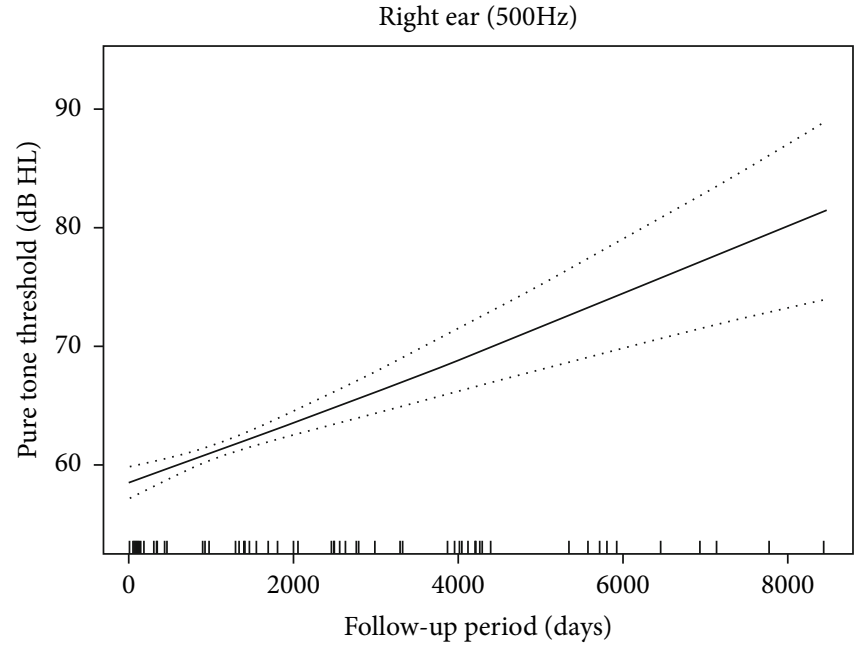

(a)

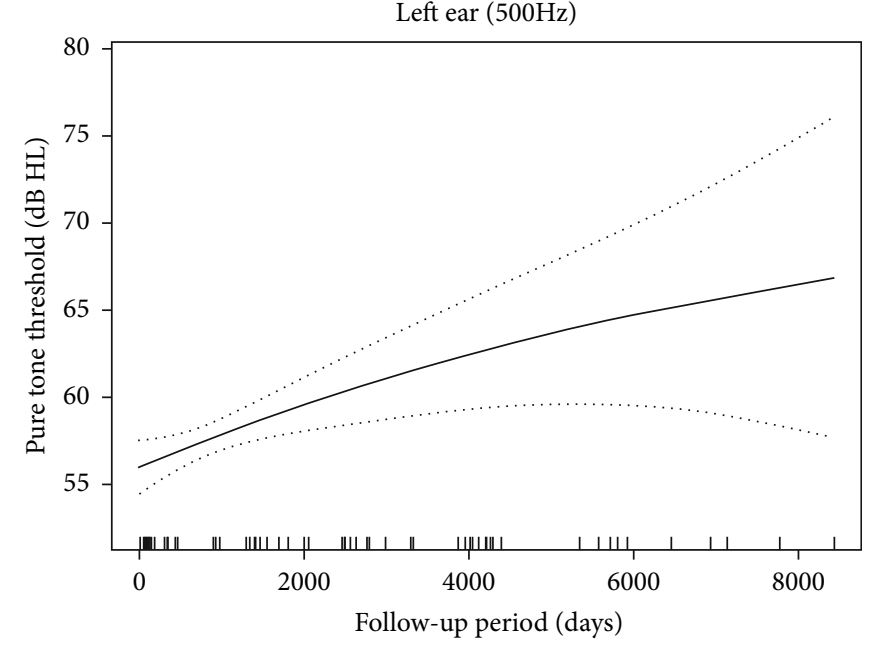

(b)

FIgURE 5: $(\mathrm{a}, \mathrm{b})$ The change of pure tone threshold of $0.5 \mathrm{kHz}$ over time in both ears of AIFM1-positive AN patients. Spline smoothing was performed using GAMM (generalized additive mixed model) to explore the change of pure tone threshold with the length of follow-up time. The solid lines represent the fitting spline. The dashed lines represent the $95 \%$ confidence intervals. The vertical axis measures the change in pure tone hearing. The rug plot provides a visual representation of the frequency distribution for follow-up time. Each individual data point is represented by a single tick mark at the appropriate location on the chosen time scale (days).
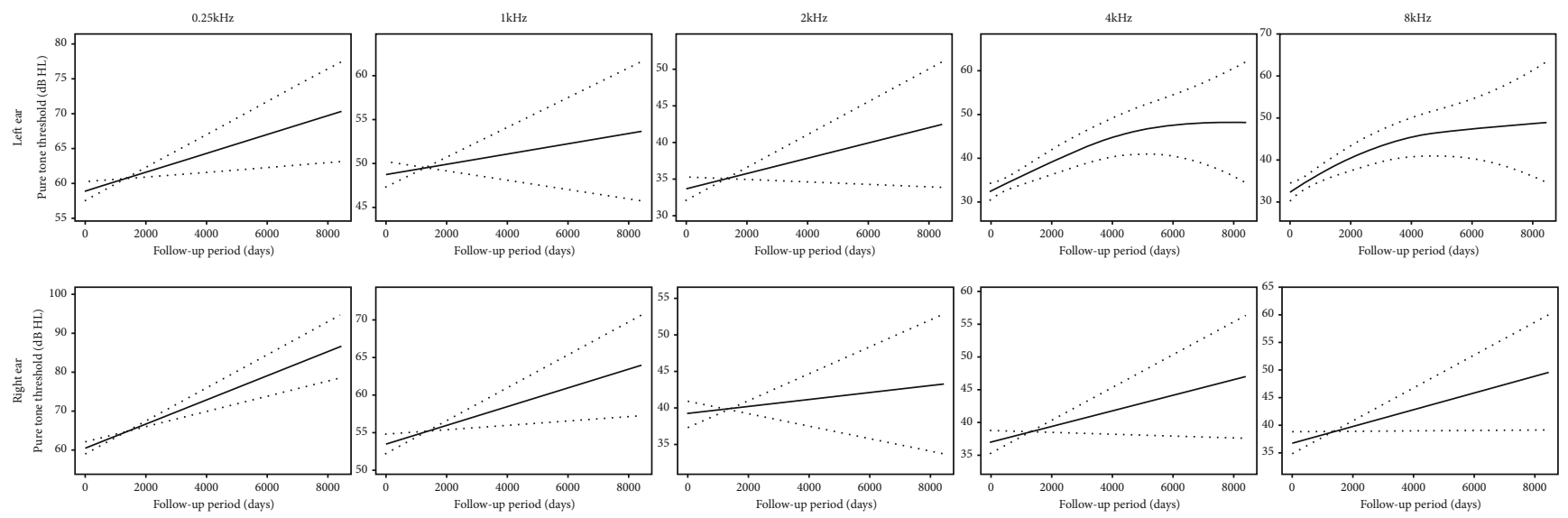

Figure 6: The change of pure tone threshold of $0.25,1,2,4$, and $8 \mathrm{kHz}$ over time in both ears of AIFM1-positive AN patients. Spline smoothing was performed using GAMM (generalized additive mixed model) to explore the change of pure tone threshold with the length of follow-up time. The solid lines represent the fitting spline. The dashed lines represent the $95 \%$ confidence intervals.

the presence or absence of CAP waves, showing that the degree of hearing loss without CAP waveform was more serious. Clinically, the detection value should be increased, and the degree of hearing loss should be comprehensively judged

Individuals with lesions affecting the auditory nerve showed poor performance with cochlear implantation, since the neural transmission was affected [2]. The audiological phenotype features of AIFM1-related AN suggested that auditory dyssynchrony progressively worsened over time. Electrophysiological examinations of the cochlear nerve indicated that lesions would be located on the auditory pathway from postsynapses to acoustic fibers [3]. Further, the diffusion-weighted MRI (dMRI) analysis techniques may contribute to the microstructure of the auditory tracts in vivo in individuals with $\mathrm{AN}[3,46]$; from the dMRI of some patients, we can see a reduction in apparent fiber density within the auditory brainstem tracts, which is consistent with the assumed pathophysiological mechanism of postsynapses to acoustic fibers (unpublished data). Thus, AN patients with AIFM1 mutation may have poor efficiency from cochlear implantation.

The molecular disease-causing mechanism of AIFM1 mutation-related hearing loss is still unclear; the mitochondrial function and the caspase-independent apoptosis to neuronal development and adult neurogenesis play a critical role in previous studies $[47,48]$. In our recent study, we found 
that the AIFM1 mutation led to decreased dimerization and further impaired mitochondrial functions, such as increase of ROS production and impairment of mitochondrial membrane potential, thereby activating caspase-independent apoptosis (unpublished data). However, the complete and clear pathogenesis of AN and the genotype-phenotype correlation need to be further clarified.

In conclusion, (1) AIFM1 is the most common genetic cause of late-onset AN, with the hotspot mutation of c.1030C > T (p.Leu344Phe). (2) In addition to the X-linked recessive inheritance pattern, the X-linked dominant inheritance pattern is another probability of AIFM1-related AN, which affects females. (3) The hearing threshold of AN patients with AIFM1 mutation tends to worsen when the follow-up period is prolonged. Phenotype features of AIFM1-related AN suggested that auditory dyssynchrony progressively worsened over time. Electrophysiological examinations of the cochlear nerve indicated that lesions would be located on the auditory pathway from postsynapses to acoustic fibers.

\section{Data Availability}

The datasets used and/or analyzed during the current study are available from the corresponding author on reasonable request.

\section{Disclosure}

The funders had no role in study design, data collection and analysis, decision to publish, or preparation of the manuscript.

\section{Conflicts of Interest}

The authors have declared that no competing interests exist.

\section{Authors' Contributions}

Hongyang Wang, Dan Bing, and Jin Li contributed equally to this study.

\section{Acknowledgments}

This work was supported by the grants of the National Natural Science Foundation of China (Nos. 81830028, 81530032, 81900950, and 81900951) and the Military Medical Technology Incubation Project for Youth (19QNP058).

\section{Supplementary Materials}

Supplementary 1. Figure S1: follow-up ECochG test of 6 cases (12 ears); there was no difference between the absolute values of -SP/AP.F

Supplementary 2. Table S1: difference value of SDS in AIFM1-positive cases. Table S2: correlation of PTA and SDS. Table S3: SDS from cases with different disease courses. Table S4: the PTA and disease course of the cases with ABR V-wave. Table S5: follow-up of the cases with ABR V-wave. Table S6: CAP waves and PTA in AIFM1-positive AN cases. Table S7: the reported variations and diseases of AIFM1.

\section{References}

[1] G. Rance, "Auditory neuropathy/dys-synchrony and its perceptual consequences," Trends in Amplification, vol. 9, no. 1, pp. 1-43, 2005.

[2] A. E. Shearer and M. R. Hansen, "Auditory synaptopathy, auditory neuropathy, and cochlear implantation," Laryngoscope Investigative Otolaryngology, vol. 4, no. 4, pp. 429-440, 2019.

[3] G. Rance and A. Starr, "Pathophysiological mechanisms and functional hearing consequences of auditory neuropathy," Brain, vol. 138, no. 11, pp. 3141-3158, 2015.

[4] V. K. C. Manchaiah, F. Zhao, A. A. Danesh, and R. Duprey, "The genetic basis of auditory neuropathy spectrum disorder (ANSD)," International Journal of Pediatric Otorhinolaryngology, vol. 75, no. 2, pp. 151-158, 2011.

[5] Q. J. Wang, Q. Z. Li, S. Q. Rao et al., “AUNX1, a novel locus responsible for $\mathrm{X}$ linked recessive auditory and peripheral neuropathy, maps to Xq23-27.3," Journal of Medical Genetics, vol. 43, no. 7, p. e33, 2005.

[6] L. Zong, J. Guan, M. Ealy et al., "Mutations in apoptosisinducing factor cause X-linked recessive auditory neuropathy spectrum disorder," Journal of Medical Genetics, vol. 52, no. 8, pp. 523-531, 2015.

[7] S. A. Susin, H. K. Lorenzo, N. Zamzami et al., "Molecular characterization of mitochondrial apoptosis-inducing factor," Nature, vol. 397, no. 6718, pp. 441-446, 1999.

[8] R. Santarelli, "Information from cochlear potentials and genetic mutations helps localize the lesion site in auditory neuropathy," Genome Medicine, vol. 2, no. 12, p. 91, 2010.

[9] T. Moser and A. Starr, "Auditory neuropathy - neural and synaptic mechanisms," Nature Reviews Neurology, vol. 12, no. 3, pp. 135-149, 2016.

[10] R. Santarelli, R. Rossi, P. Scimemi et al., "OPA1-related auditory neuropathy: site of lesion and outcome of cochlear implantation,” Brain, vol. 138, no. 3, pp. 563-576, 2015.

[11] W. Zhang, S. M. Kim, W. Wang et al., "Cochlear gene therapy for sensorineural hearing loss: current status and major remaining hurdles for translational success," Frontiers in Molecular Neuroscience, vol. 11, 2018.

[12] B. Pan, C. Askew, A. Galvin et al., "Gene therapy restores auditory and vestibular function in a mouse model of Usher syndrome type 1c," Nature Biotechnology, vol. 35, no. 3, pp. 264-272, 2017.

[13] R. Sacheli, L. Delacroix, P. Vandenackerveken, L. Nguyen, and B. Malgrange, "Gene transfer in inner ear cells: a challenging race," Gene Therapy, vol. 20, no. 3, pp. 237-247, 2013.

[14] S. Richards, on behalf of the ACMG Laboratory Quality Assurance Committee, N. Aziz et al., "Standards and guidelines for the interpretation of sequence variants: a joint consensus recommendation of the American College of Medical Genetics and Genomics and the Association for Molecular Pathology," Genetics in Medicine, vol. 17, no. 5, pp. 405-423, 2015.

[15] T. Kawarai, H. Yamazaki, K. Yamakami et al., "A novel AIFM1 missense mutation in a Japanese patient with ataxic sensory neuronopathy and hearing impairment," Journal of the Neurological Sciences, vol. 409, p. 116584, 2020.

[16] P. Bogdanova-Mihaylova, M. D. Alexander, R. P. Murphy et al., "Clinical spectrum of AIFM1-associated disease in an Irish family, from mild neuropathy to severe cerebellar ataxia with colour blindness," Journal of the Peripheral Nervous System, vol. 24, no. 4, pp. 348-353, 2019. 
[17] B. Wang, X. Li, J. Wang et al., "A novel_AIFM1_mutation in a Chinese family with X-linked Charcot -Marie- Tooth disease type 4," Neuromuscular Disorders, vol. 28, no. 8, pp. 652659, 2018.

[18] P. Sancho, A. Sánchez-Monteagudo, A. Collado et al., "A newly distal hereditary motor neuropathy caused by a rare AIFM1 mutation," Neurogenetics, vol. 18, no. 4, pp. 245-250, 2017.

[19] S. U. Morton, S. P. Prabhu, L. HGW et al., "AIFM1mutation presenting with fatal encephalomyopathy and mitochondrial disease in an infant.," Molecular Case Studies, vol. 3, no. 2, p. a001560, 2017.

[20] N. Miyake, N. I. Wolf, F. K. Cayami et al., "X-linked hypomyelination with spondylometaphyseal dysplasia (H-SMD) associated with mutations in AIFM1," Neurogenetics, vol. 18, no. 4, pp. 185-194, 2017.

[21] H. Mierzewska, M. Rydzanicz, T. Biegański et al., "Spondyloepimetaphyseal dysplasia with neurodegeneration associated with AIFM1 mutation - a novel phenotype of the mitochondrial disease," Clinical Genetics, vol. 91, no. 1, pp. 30-37, 2017.

[22] B. Hu, M. Wang, R. Castoro et al., "A novel missense mutation in AIFM1 results in axonal polyneuropathy and misassembly of OXPHOS complexes," European Journal of Neurology, vol. 24, no. 12, pp. 1499-1506, 2017.

[23] D. Diodato, G. Tasca, D. Verrigni et al., "A novel_AIFM1_ mutation expands the phenotype to an infantile motor neuron disease," European Journal of Human Genetics, vol. 24, no. 3, pp. 463-466, 2016.

[24] M. Kettwig, M. Schubach, F. A. Zimmermann et al., "From ventriculomegaly to severe muscular atrophy: expansion of the clinical spectrum related to mutations in AIFM1," Mitochondrion, vol. 21, pp. 12-18, 2015.

[25] A. Ardissone, G. Piscosquito, A. Legati et al., "A slowly progressive mitochondrial encephalomyopathy widens the spectrum of AIFM1 disorders," Neurology, vol. 84, no. 21, pp. 2193-2195, 2015.

[26] C. Rinaldi, C. Grunseich, I. F. Sevrioukova et al., "Cowchock syndrome is associated with a mutation in apoptosisinducing factor," The American Journal of Human Genetics, vol. 91, no. 6, pp. 1095-1102, 2012.

[27] I. Berger, Z. Ben-Neriah, T. Dor-Wolman et al., "Early prenatal ventriculomegaly due to an AIFM1 mutation identified by linkage analysis and whole exome sequencing," Molecular Genetics and Metabolism, vol. 104, no. 4, pp. 517-520, 2011.

[28] D. Ghezzi, I. Sevrioukova, F. Invernizzi et al., "Severe X-linked mitochondrial encephalomyopathy associated with a mutation in apoptosis-inducing factor," The American Journal of Human Genetics, vol. 86, no. 4, pp. 639-649, 2010.

[29] Y. Liu, J. Qi, X. Chen et al., "Critical role of spectrin in hearing development and deafness," Science Advances, vol. 5, no. 4, p. eaav7803, 2019.

[30] L. Liu, Y. Chen, J. Qi et al., "Wnt activation protects against neomycin-induced hair cell damage in the mouse cochlea," Cell Death \& Disease, vol. 7, no. 3, p. e2136, 2016.

[31] Z. He, L. Guo, Y. Shu et al., "Autophagy protects auditory hair cells against neomycin-induced damage," Autophagy, vol. 13, no. 11, pp. 1884-1904, 2017.

[32] C. Zhu, C. Cheng, Y. Wang et al., "Loss of ARHGEF6 causes hair cell stereocilia deficits and hearing loss in mice," Frontiers in Molecular Neuroscience, vol. 11, 2018.
[33] W. Liu, X. Xu, Z. Fan et al., "Wnt signaling activates TP53induced glycolysis and apoptosis regulator and protects against cisplatin-induced spiral ganglion neuron damage in the mouse cochlea," Antioxidants \& Redox Signaling, vol. 30, no. 11, pp. 1389-1410, 2019.

[34] Z. H. He, S. Y. Zou, M. Li et al., "The nuclear transcription factor FoxG1 affects the sensitivity of mimetic aging hair cells to inflammation by regulating autophagy pathways," Redox Biology, vol. 28, p. 101364, 2020.

[35] S. Zhang, Y. Zhang, Y. Dong et al., "Knockdown of Foxg1 in supporting cells increases the trans-differentiation of supporting cells into hair cells in the neonatal mouse cochlea," Cellular and Molecular Life Sciences, vol. 77, no. 7, pp. 1401-1419, 2020.

[36] F. Tan, C. Chu, J. Qi et al., "AAV-ie enables safe and efficient gene transfer to inner ear cells," Nature Communications, vol. 10, no. 1, p. 3733, 2019.

[37] C. Cheng, Y. Wang, L. Guo et al., "Age-related transcriptome changes in Sox $2+$ supporting cells in the mouse cochlea," Stem Cell Research \& Therapy, vol. 10, no. 1, 2019.

[38] W. Yan, W. Liu, J. Qi et al., “A three-dimensional culture system with Matrigel promotes purified spiral ganglion neuron survival and function in vitro," Molecular Neurobiology, vol. 55, no. 3, pp. 2070-2084, 2018.

[39] X. Lu, S. Sun, J. Qi et al., "Bmil regulates the proliferation of cochlear supporting cells via the canonical Wnt signaling pathway," Molecular Neurobiology, vol. 54, no. 2, pp. 1326-1339, 2017.

[40] T. Wang, R. Chai, G. S. Kim et al., "Lgr5+ cells regenerate hair cells via proliferation and direct transdifferentiation in damaged neonatal mouse utricle," Nature Communications, vol. 6, no. 1, 2015.

[41] B. C. Cox, R. Chai, A. Lenoir et al., "Spontaneous hair cell regeneration in the neonatal mouse cochlea in vivo," Development, vol. 141, no. 4, pp. 816-829, 2014.

[42] Q. J. Zhang, B. Han, L. Lan et al., "High frequency of OTOF mutations in Chinese infants with congenital auditory neuropathy spectrum disorder," Clinical Genetics, vol. 90, no. 3, pp. 238-246, 2016.

[43] H. Wu, Y. Feng, L. Jiang et al., "Application of a new genetic deafness microarray for detecting mutations in the deaf in China," PLoS One, vol. 11, no. 3, article e0151909, 2016.

[44] D. P. Wynne, F. G. Zeng, S. Bhatt, H. J. Michalewski, A. Dimitrijevic, and A. Starr, "Loudness adaptation accompanying ribbon synapse and auditory nerve disorders," Brain, vol. 136, no. 5, pp. 1626-1638, 2013.

[45] W. P. Gibson, "The clinical uses of electrocochleography," Frontiers in Neuroscience, vol. 11, p. 274, 2017.

[46] J. Zanin, T. Dhollander, S. Farquharson, G. Rance, A. Connelly, and B. A. Nayagam, "Review: using diffusion-weighted magnetic resonance imaging techniques to explore the microstructure and connectivity of subcortical white matter tracts in the human auditory system," Hearing Research, vol. 377, pp. 1-11, 2019.

[47] C. Reinhardt, G. Arena, K. Nedara et al., "AIF meets the CHCHD4/Mia40-dependent mitochondrial import pathway," Biochimica et Biophysica Acta (BBA) - Molecular Basis of Disease, vol. 1866, no. 6, p. 165746, 2020.

[48] E. Hangen, O. Féraud, S. Lachkar et al., "Interaction between AIF and CHCHD4 regulates respiratory chain biogenesis," Molecular Cell, vol. 58, no. 6, pp. 1001-1014, 2015. 